\title{
Mining Semantic Classes of Image Databases
}

\author{
Zaher Al Aghbari
}

\begin{abstract}
This paper demonstrates an approach to mine semantic classes of image regions (e.g. sky, snow, rocks, etc.). Particularly, classify image regions based on their semantics using a support vector machines (SVMs). The SVMs learns the semantics of specified classes from specific low-level feature of the test image regions. Image regions are, first, segmented using a hill-climbing approach. Then, those regions are classified by the SVMs. Such semantic classification allows the implementation of intuitive query interface. In these experiments, the high precision of semantic classification justifies the feasibility of our approach.
\end{abstract}

Index Terms-Image mining, image classification, hill-climbing, semantic classification.

\section{INTRODUCTION}

Content-based image/video classification and retrieval systems have been the subject of many research works in recent years [1]. Although these works enjoy the advantage of being fully automatic, they are hard to use by non-expert users due to the semantic gap that exists between user needs and the low-level features descriptions of images. To bridge this semantic gap, we need to classify image regions based on their semantics and then build an index that utilizes these semantics. As a result, non-expert users can intuitively formulate queries to search for desired images.

Automatic extraction of semantic elements of images is not yet reliable as opposed to the low-level features [2]. However, there have been some research works to identify (label) image elements. The work in [3] manually labels the image element (called visual keyword) from samples of visual content domain. Then, every image in the database is compared against those visual keywords to detect the specific visual keywords of the image. The approach in [4] proposes a probabilistic approach to label small areas of one set of images as either man-made images or natural, and the local areas of another set were labeled as either inside or outside. Another approach [5] proposes a Bayesian framework for classifying vacation images. Further, based on the k-nearest neighbor classifier, the work in [6] classifies internet images into photographical versus synthetic. The work in [7] represented the image colors as time series and used the SAX method to classify the images.

A different approach to organize the images into semantic categories is shown in [8], in which training sample regions of images are divided by a neural network into a one-level set of [9] classes (e.g. Brick, Cloud, Grass, etc.) rather than hierarchal classes. A different classifier was created for every

Manuscript received January 24, 2014; revised April 1, 2014.

Zaher Al Aghbari is with the Department of Computer Science, University of Sharjah, UAE (e-mail: zaher@sharjah.ac.ae). collection of images. Similarly, the approach in [9] classifies the image regions into 10 flat classes (e.g. sky, water, night, etc.).

In this paper, we propose to extend the approaches of the automatic hierarchal classification of image regions into a more detailed classification hierarchy based on the semantics of the region content. The paper also demonstrates that we can reliably classify regions of outdoor images into semantic classes, which humans already innately understand. We use a hill-climbing method [10] to segment images. Learning the semantics of a predefined hierarchy of classes is performed using support vector machines (SVMs). The SVMs is applied to heterogeneous features of the training sample regions that represent every class. Then, the created "conservative" classifiers P(classified | not Member Of Class) achieves good class separation under the constraint that each region belongs to only one, or none, of the classes.

In the rest of this paper, we discuss image segmentation and the extraction heterogeneous feature extraction from image regions in Section II. Classification of image regions is presented in Section III. Our evaluation and experiment results are presented in Section IV. Finally, we conclude the paper in Section V.

\section{FeAture EXtraction}

Image segmentation is a process of decomposing an image into coherent regions. We map the image into the HSV color space.

\section{A. Hill-Climbing Algorithm}

The Hill-Climbing algorithm [10] detects the peaks of clusters in the global three-dimensional color histogram of an image. The hill-climbing algorithm is summarized as follows:

1) Compute the global 3-dimensional HSV color histogram of the image.

2) Start at a non-zero bin of the color histogram and make uphill moves until reaching a peak (local maximum) as follows:

- Compare the number of pixels of the current histogram bin with the number of pixels of neighboring bins. Note that the feature space is three-dimensional, thus each bin in the color histogram has $3^{d}-1=26$ neighbors, where $d$ is the dimensionality of the feature space.

- If the neighboring bins have different numbers of pixels, the algorithm makes an uphill move towards the neighboring bin with larger number of pixels.

- If the immediate neighboring bins have the same number of pixels, the algorithm checks the next neighboring bins, and so on, until a set of neighboring bins with different number of pixels are found. Then, an 
uphill move is made towards the bin with larger number of pixels.

- The uphill climbing is continued (repeat steps 2.a-2.c) until reaching a bin from where there is no possible uphill movement. That is the case when the neighboring bins have smaller numbers of pixels than the current bin. Hence, the current bin is identified as a peak.

3) Select another unclimbed bin as a starting bin and perform step 2 to find another peak. This step is continued until all non-zero bins of the color histogram are climbed (associated with a peak).

4) The identified peaks represent the initial number of clusters of the input image; thus these peaks are saved.

5) Finally, neighboring pixels that lead to the same peak are grouped together, that is associating every pixel with one of the identified peaks. Consequently, the segments of the input image are formed.

Although, we used a global color histogram to find the peaks, step 5 takes into account the spatial information of the pixels when forming the segments, i.e. only spatially close (neighboring) pixels that lead to the same peak are grouped into one segment.

\section{B. Region Features}

Color Histogram: A good color space is one in which the perceived color differences should correspond to their Euclidean distances in this chosen color space. The HSV color model satisfies this property [4]. The color histogram is represented by a 24 -dimensional vector.

Edge Direction Histogram: Edge detection is often the first step in image processing since a lot of information can be obtained from the edges. There are many different types of edge detector operators. We use the popular Canny edge detector [11]. The Canny edge detector is a first derivative Gaussian. It optimizes the trade-off between noise suppression and localization.

The domain of edge direction angle is [0 - 360], which is divided into 72 bins to obtain a histogram. The direction of an edge is computed as:

$$
\theta=\arctan \left(X_{i, j} / Y_{i, j}\right) \quad \text { for } e_{i, j}=1
$$

Higher-Order Autocorrelation for Edge. The higher order autocorrelation features are the primitive edge features that are shift-invariant (irrelevant to where the objects are located in the image), which is a useful property in image querying [11]. Before extracting these features, an edge image is constructed using the Canny method.

Let an image plane be $P$ and a function $I(r)$ represents an image intensity function on the retinal plane $P$ such that $r \in P$. That is, $r$ is the image coordinate vector. A shift (translation) of $I(r)$ within $P$ is represented by $I\left(r+a_{i}\right)$, where $a_{i}$ is the displacement vector. Therefore, the $N$ th-order autocorrelation functions with $N$ displacements $a_{1}, \ldots, a_{N}$ are defined by

$$
R^{N}\left(a_{1}, \ldots, a_{N}\right)=\sum_{P} I(r) I\left(r+a_{1}\right) \ldots I\left(r+a_{N}\right)
$$

Here, we limit the order $N$ to 2 . Also, the range of displacements is limited to within a local $3 \times 3$ window, of which the center is the reference local point. By eliminating the displacements that are equivalent by shift, the number of unique patterns is reduced to 20 . Using these mask patterns, the feature vector $f^{v}$ that contains the higher order autocorrelation functions is defined as follows: $f^{\prime}=f_{1}, \ldots$, $f_{20}$.

$$
\begin{aligned}
& f_{1}=\sum_{x} \sum_{y} I(x, y) I(x-1, y) I(x+1, y) \\
& f_{20}=\sum_{x} \sum_{y} I(x, y) I(x-1, y-1) I(x+1, y-1)
\end{aligned}
$$

Dual-Tree Complex Wavelet Transform. The a dual-tree complex wavelet transform (DT CWT) is proposed by [12] to have directional selectivity and obtain shift invariance. Since DT CWT uses complex coefficient filters to have directional selectivity and uses two parallel undecimated trees of filters to have shift invariance. The subsampled outputs of the row filters and their complex conjugates form six bandpass images, three in each quadrant, and these subimages are strongly oriented at: $-15^{\circ},+15^{\circ},-45^{\circ}$, $+45^{\circ},-75^{\circ},+75^{\circ}$. These orientations are obtained since complex filters can separate positive and negative frequencies in both horizontal and vertical directions. Since the redundancy is $4: 1$ (for an image), our feature vector has 24 values (24-dimensional), where each value is averaged over the whole subsampled image.

\section{REGION CLASSIFICATION}

To classify image regions into semantic classes, which humans have an innate understanding, we first manually defined a hierarchy that reflects the semantics in the Nature images, as shown in Fig. 1.

\section{A. Learning Stage}

Learning the semantics of each class is performed using SVMs based on heterogeneous features of training sample regions of each class. We built the "conservative" SVMs classifiers:

$$
\text { P(classified | notMemberOfClass) }
$$

These binary classifiers achieve good class separation under the constraint that each region belongs to only one, or none, of the classes. Due to the fact that different semantic classes may have similar visual features (such as OceanSunset and SkySunset classes), it is difficult to build a precise binary classifier using the class organization of Fig. 1. After extensive experimentation with our system, we found that for the purpose of identifying the image regions, it is essential to reorganize the defined classes as shown in Fig. 2. This organization reflects the similarity/dissimilarity of visual features of the semantic classes. Hence, while the organization of Fig. 1 is easier to understand by humans and thus used to index and retrieve images, we use the organization of Fig. 2 to identify the members of the different classes.

Using the organization of Fig. 2, the SVMs binary classifier determines whether a certain image region is a member of some class $C_{i}$ or not. If the image region is 
identified as a member of class $C_{i}$ it is then mapped into $C_{i}$ in the semantic class hierarchy of Fig. 1 For example, Fig. 3 shows a binary SVMs classifier P(Sand\&Rock | notMemberOf Sand\&Rock) that differentiates regions that belong to Sand\&Rock collection from others and then the regions of the Sand\&Rock collection undergo further identification test to be classified into the Sand class by the $\mathrm{P}($ Sand $\mid$ notMemberOf Sand) classifier or into the Rock class by the P(Rock | notMemberOf Rock) classifier. Then, members of the Sand and Rock classes are mapped into their respective classes on the semantic class hierarchy of Fig. 1.

For each classifier in Fig. 2, we prepared a training set of sample image regions. We selected 800 region samples to train the binary SVMs classifiers; on the average, 60 training samples per class. Note that each classifier distinguishes members of its class based on visual features that may not be the same as those of other classifiers as shown on Fig. 2.

\section{B. Classification Stage}

After training the SVMs, binary classifiers that can classify image regions based on their semantics are created. Then, we use these binary classifiers to classify our database of image regions leading to the classification shown in Fig. 1. As discussed above, we use the organization of Fig. 2 to determine the class of an input image region and this image region is mapped into its class in the semantic class hierarchy (Fig. 1). In the semantic class hierarchy, all scenery regions are classified into Nature regions or Artificial regions. Since in this paper we concentrate on Nature regions, thus we further classify the Nature regions into 3 subclasses: Water, Sky and Land. Each one of these subclasses is further divided into subsubclasses. The Water subclass is divided into OceanSunset, BlueOcean and Waterfall. Next, the Sky subclass is divided into Night, SkySunset, Cloud and BlueSky. Then, the Land subclass is divided into Rocks, Vegetation, Snow and Sand. Also, the Vegetation subsubclass is further divided into GreenForest and RedForest.

Using the organization of Fig. 2, the SVMs binary classifier determines whether a certain image region is a member of some class $C_{i}$ or not. If the image region is identified as a member of class $C_{i}$ it is then mapped into $C_{i}$ in the semantic class hierarchy of Fig. 1. For example, Fig. 3 shows a binary SVMs classifier that maps members of the Sand and Rock classes into their respective classes on the semantic class hierarchy of Fig. 1.

\section{EXPERIMENTS}

We used 6000 images in the experiments that were selected from two collections. On the average, 5 regions were produced from each image as a result of segmentation. Thus, we have approximately a database of 30000 regions from which we selected 770 regions for training the SVMs. That is about 60 images per class.

To classify the image regions, we tried different grouping of classes and different features before we finally decided on the grouping and features in Fig. 2, which gives the best classification precision. Our goal in the different grouping is to have high classification precision at the top levels since lower level classifications cannot exceed that of the top level.
At first, we performed the classification Nature regions vs. Artificial regions using different features as shown in Table I. Notice that it is not possible to classify these two classes using color feature since regions with similar color exist in both classes. However, edge features show good classification results since edges in Artificial regions tend to be more straight than in Nature regions. We chose the CWT feature since it gives slightly better precision than the EDH feature.

TABLE I: CLASSIFICATION OF NATURE REGIONS VS. ARTIFICIAL REGIONS

\begin{tabular}{ccc}
\hline \hline & Nature & Artificial \\
\hline EDH & 90.7 & 90.7 \\
CWT & 90.8 & 91.0 \\
HAC & Not possible & Not possible \\
\hline \hline
\end{tabular}

Then, we tried to group the Nature image regions based on their semantics into 3 classes as shown in Fig. 1. Classifications with different features were performed and the best classification precision was attained using the CWT feature and summarized in Table II. Note that the precision values are relatively low and thus lower level classifications will not exceed the precision values in Table II. Therefore, we tried different regroupings of the classes and performed the experiments after each regrouping. Finally, the best classification results were achieved for the grouping shown in Fig. 2 whose precision values are summarized in Table III. For this level, the best classification results was attained using the color features due to the nature of grouping, which reflect the similarity of color feature between subclasses of the same group (subtree in Fig. 2). Clearly, the classification precision results are high and thus the groupings of Table III are chosen for the level below the Nature class.

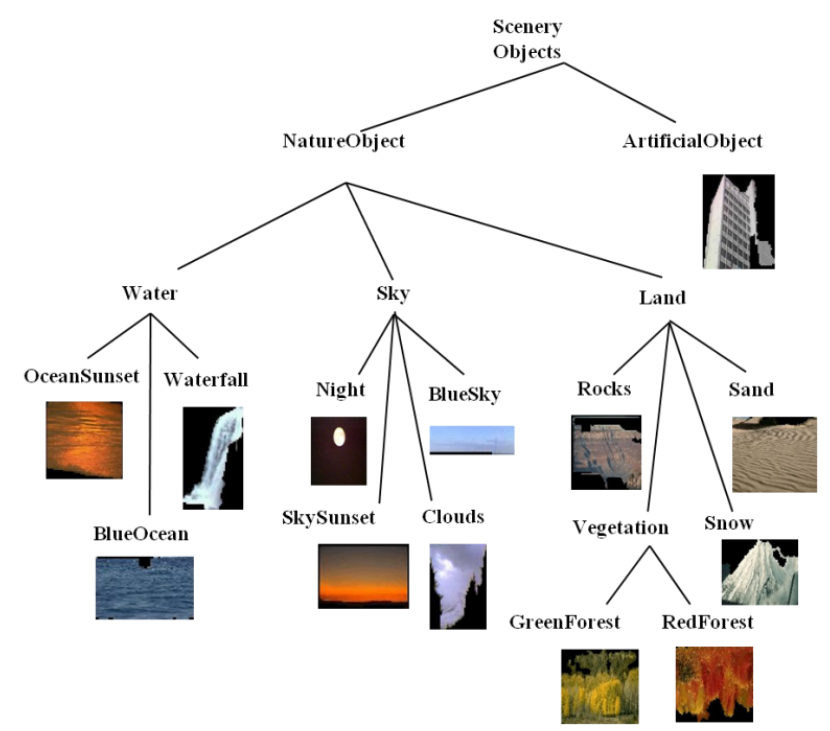

Fig. 1. A class hierarchy that reflects the semantics of nature image regions.

As shown in Fig. 2, the Blue\&White, Sand\&Rock and Sunset classes are further divided into subclasses. The Blue\&White class was divided into 5 subclasses as shown in Fig. 2. The precision results are shown in Table IV. Note that different subclasses are better distinguished by different features from the other subclasses within the Blue\&White class. Thus, we selected the features that give the best precision classification: EDH for BlueSky, HAC for Cloud, CWT for Snow, EDH for Waterfall and EDH for BlueOcean. 


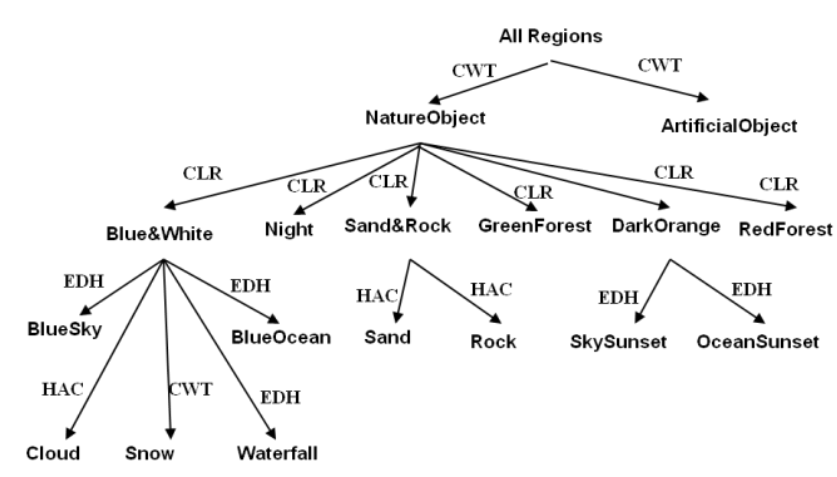

Fig. 2. A class organization used by the SVMs to distinguish the members of a class from others. Where CLR=color, $\mathrm{CWT}=$ dual-tree complex wavelet transform, $\mathrm{EDH}=$ edge direction histogram, and $\mathrm{HAC}=$ higher-order autocorrelation edge feature.

Similarly, each of the Sand\&Rock and Sunset classes were divided into 2 subclasses and the precision values of the classification experiments are summarized in Table $\mathrm{V}$ and Table VI, respectively. Note that the HAC feature leads to the best classification precision for both subclasses Sand and Rock as shown in Table V. For the Rock subclass, the CWT feature could also be used for classification since its precision is equal to that of the HAC feature. On the other hand, the EDH feature lead to the best classification precision for the SkySunset and OceanSunset subclasses as shown in Table VI.

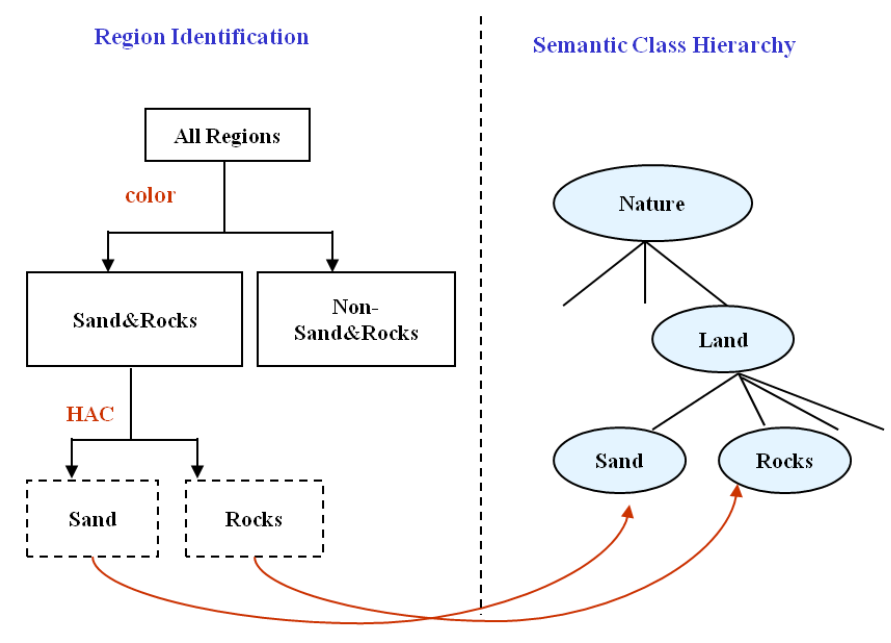

Fig. 3. Mapping identified regions into the semantic class hierarchy.

TABLE II: CLASSIFICATION OF SEMANTIC GROUPS USING CWT

\begin{tabular}{lcl}
\hline \multicolumn{1}{c}{ Class Name } & Precision & \multicolumn{1}{c}{ Subclasses } \\
\hline Sky & 83.3 & Night, SunsetSky, Cloud, BlueSky \\
Land & 78.6 & $\begin{array}{l}\text { Rock, Sand, Snow, GreenForest, } \\
\text { RedForest }\end{array}$ \\
Water & 78.1 & OceanSunset, BlueOcean, Waterfall \\
\hline \hline
\end{tabular}

TABLE III: CLASSIFICATION AFTER REGOUPING CLASSES AS SHOWN IN FIG. 2 USING COLOR

\begin{tabular}{lcl}
\hline Class Name & Precision & \multicolumn{1}{c}{ Subclasses } \\
\hline Blue\&White & 93.2 & $\begin{array}{l}\text { BlueSky, Cloud, Snow, Waterfall, } \\
\text { BlueOcean }\end{array}$ \\
Night & 95.8 & \\
Sand\&Rock & 91.4 & Sand, Rock \\
GreenForest & 97.2 & \\
Sunset & 91.8 & SkySunset, OceanSunset \\
RedForest & 95.7 & \\
\hline \hline
\end{tabular}

TABLE IV: PRECISION VALUES OF CLASSIFYING THE SUBCLASSES OF THE BLUE\&WHITE CLASS

\begin{tabular}{c|ccccc}
\hline \hline \multirow{2}{*}{ Feature } & \multicolumn{5}{|c}{ Blue\&White } \\
\cline { 2 - 6 } & BlueSky & Cloud & Snow & Waterfall & BlueOcean \\
\hline EDH & 89.6 & 84.0 & 91.2 & 88.1 & 85.9 \\
CWT & 80.6 & 83.4 & 93.3 & 83.3 & 80.3 \\
HAC & 80.6 & 84.4 & 92.1 & 84.0 & 79.2 \\
\hline \hline
\end{tabular}

Similarly, each of the Sand\&Rock and Sunset classes were divided into 2 subclasses and the precision values of the classification experiments are summarized in Tables $\mathrm{V}$ and VI, respectively. Note that the HAC feature leads to the best classification precision for both subclasses Sand and Rock as shown in Table V. For the Rock subclass, the CWT feature could also be used for classification since its precision is equal to that of the HAC feature. On the other hand, the EDH feature lead to the best classification precision for the SkySunset and OceanSunset subclasses as shown in Table VI.

TABLE V: PRECISION VALUES OF ClASSIFYING THE SUBCLASSES OF THE

\begin{tabular}{c|cc}
\multicolumn{2}{c}{ SAND\&ROCK CLASS } \\
\hline \hline \multirow{2}{*}{ Feature } & \multicolumn{2}{|c}{ Sand\&Rock } \\
\cline { 2 - 3 } & Sand & Rock \\
\hline EDH & 86.7 & 85.3 \\
CWT & 85.3 & 89.3 \\
HAC & 89.3 & 89.3 \\
\hline \hline
\end{tabular}

TABLE VI: PRECISION VALUES OF CLASSIFYING THE SUBCLASSES OF THE

\begin{tabular}{c|cc}
\multicolumn{2}{c}{ SUNSET CLASS } \\
\hline \multirow{2}{*}{ Feature } & \multicolumn{2}{|c}{ Sunset } \\
\cline { 2 - 3 } & SkySunset & OceanSunset \\
\hline EDH & 87.4 & 86.7 \\
CWT & 86.7 & 86.0 \\
HAC & 83.9 & 83.5 \\
\hline
\end{tabular}

Although we used the organization of Fig. 2 to determine the class of an input image region, this image region is then mapped into its class in the semantic class hierarchy (Fig. 1). Then, we represented an image by the classes, in which its image regions are classified.

\section{CONCLUSION}

This paper presented an approach to classify image regions based on their semantics rather than their low-level features. The pre-defined semantic class hierarchy reflects the semantics in the Nature image regions that humans have an innate understanding. Hence, flexible and intuitive query compositions can be based on such semantic hierarchy. As each image is represented by the classes in which its regions were classified into, these semantic classes (labels of an image) are a high-level descriptor that can be easily understood by a novice user. The use of the binary SVMs classifiers that classify image regions using different features at different levels in the hierarchy were the main reasons behind the high classification precision that we achieved in our experiments. Currently, we are looking into adding more classifiers to include more classes into the system. A final goal is to generate semantic indices into image databases.

\section{REFERENCES}

[1] W. Wang, Y. Song, and A. Zhang, "Semantic-Based image retrieval by region saliency," presented at Int'l Conf. On Image and Video Retrieval, July 2002. 
[2] Z. A. Aghbari, K. Kaneko, and A. Makinouchi, "New indexing method for content-based video retrieval and clustering for MPEG video database," in Proc. International Symposium on Digital Media Information Base (DMIB'97), Nov. 1997, pp. 140-149.

[3] R. Weber, H.-J. Schek, and S. Blott, "A quantitative analysis and performance study for similarity-search methods in high-dimensional spaces," presented at 24th VLDB, USA, 1998.

[4] P. Mulhem and J.-H. Lim, "Symbolic photograph content-based retrieval," presented at ACM Int'l Conf. On Information and Knowledge Management (CIKM), USA, Nov. 2002.

[5] B. Bradshaw, "Semantic based image retrieval: A probabilistic approach," ACM Multimedia, Oct. 2000.

[6] A. Vailaya, A. Jain, M. Figueiredo, and H. J. Zhang, "Content-Based hierarchial classification of vacation images," presented at IEEE Int'l Conf. On Multimedia Computing and Systems, Jun. 1999.

[7] T. Gevers, F. Aldershoff, and A. W. M. Smeulders, "Classification of images on internet by visual and textual information," in Proc. SPIE Internet Imaging, 1999, vol. 3964.

[8] Z. A. Aghbari, "Effective image mining by representing color histograms as time series," International Journal of Advanced Computational Intelligence and Intelligent Informatics, vol. 13, no.2, pp. 109-114, 2009.

[9] C. P. Town and D. Sinclair, "Content based image retrieval using semantic visual categories," Technical Report TR2000-14, AT\&T Laboratories Cambridge, 2000.

[10] Z. A. Aghbari and R. Al-Haj, "Hill-Manipulation: An effective algorithm for color image segmentation," Elsevier International
Journal on Image and Vision Computing, vol. 24, no. 8, pp. 894-903, Aug. 2006.

[11] J. Canny, "A computational approach to edge detection," IEEE Trans. on Pattern Analysis and Machine Intelligence, vol. 8, pp. 679-698, 1986.

[12] M. Kubo, Z. A. Aghbari, K.-S. Oh, and A. Makinouchi, "Content-Based image retrieval technique using wavelet-based shift and brightness invariant edge feature," International Journal on Wavelets, Multiresolution and Information Processing (IJWMIP), vol. 1, no. 2, pp. 163-178, 2003.

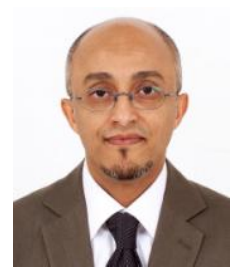

Zaher Al Aghbari received his B.Sc degree from the Florida Institute of Technology, Melbourne, USA, in 1987, and the M.Sc. and Ph.D. degrees in computer science from Kyushu University, Fukuoka, Japan, in 1998 and 2001, respectively. He was with at the Department of Intelligent Systems, Kyushu University, Japan, from 2001 to 2003. Since 2003 he was with the Department of Computer Science, University of Sharjah, UAE. He was the head of the Department of Computer Science from 2008 to 2012. Currently, he is a professor of databases and data mining in the Department of Computer Science, College of Sciences, University of Sharjah. His research interests include data mining, multidimensional indexing, multimedia databases, distributed indexing, data streams management, social networks, WSN data mining, and Arabic handwritten text retrieval. 